\title{
Bayesian estimation based on generalized order statistics from exponentiated Weibull Poisson model
}

\author{
Abd-Elfattah A. M, Amal S. Hassan, Said G. Nassr * \\ Department of Mathematical Statistical, Institute of Statistical Studies \& Research, Cairo University, Egypt \\ *Corresponding authorE-mail:dr.saidstat@gmail.com
}

Copyright $(\odot) 2015$ Abd-Elfattah, et al. This is an open access article distributed under the Creative Commons Attribution License, which permits unrestricted use, distribution, and reproduction in any medium, provided the original work is properly cited.

\begin{abstract}
In this research paper, the estimation of the unknown parameters for the exponentiated Weibull Poisson distribution using the concept of generalized order statistics is investigated from Bayesian approach. The squared error, LINEX and general entropy loss functions are considered for Bayesian computation. Bayes estimates based on Progressively type II censored and the joint density function of ordinary order statistics are considered as special cases of generalized order statistics. Finally simulation study is conducted for illustrative purposes.
\end{abstract}

Keywords: Generalized Order Statistics; Exponentiated Weibull Poisson Distribution; Bayesian Method; Progressively Type-II Censored; Joint Density Function of Ordinary Order Statistics.

\section{Introduction}

Order statistics and record values play an important role in statistics and its applications in reliability theory and life testing. Their distributional and stochastic properties have been studied extensively in the literature. However, they can be considered as special cases of generalized order statistics (GOS) that have been introduced and extensively studied by Kamps [17]. Its enable a unified approach to several models of ordered random variables such as ordinary order statistics, record values, sequential order statistics, and progressively type II censored statistics.

The random variables $X(1, n, m, k), X(2, n, m, k), \ldots, X(n, n, m, k)$ are called GOS if their joint probability density function is given by

$$
\begin{array}{r}
f_{X(1, n, m, k), X(2, n, m, k), \ldots, X(n, n, m, k)}\left(x_{(1)}, x_{(2)}, \ldots, x_{(n)}\right)=C_{n-1}\left[\prod_{i=1}^{n-1}\left(\bar{F}\left(x_{(i)}\right)\right)^{m_{i}} f\left(x_{(i)}\right)\right]\left[\bar{F}\left(x_{(n)}\right)\right]^{k-1} f\left(x_{(n)}\right) \\
\quad \text { for } F^{-1}(0)<x_{(1)} \leq x_{(2)} \leq \cdots \leq x_{(n)}<F^{-1}
\end{array}
$$

where, $C_{n-1}=k\left(\prod_{j=1}^{n-1} \gamma_{j}\right), \gamma_{j}=k+n-j+\prod_{i=j}^{n-1} m_{i}$, and $\bar{F}(x)=1-F(x)$.

For suitable choices of the parameters, GOS reduced to the well-known ordered random variable. If $m_{1}=m_{2}=\cdots=$ $m_{n-1}=0$ and $k=1$, then (1) reduced to the joint density function of ordinary order statistics. If $m_{i}=R_{i}, i=$ $1,2, \ldots, n-1$ and $k=\gamma_{n}=R_{n}+1$, then (1) reduced to the progressive type II censored data.

The statistical properties and the estimation problems based on generalized order statistics for some life time distributions are studied by several researchers. Ahsanallah [5] studied the distribution properties of GOS for a uniform distribution. Ahsanallah [6] studied some distributional properties of GOS for the two parameter exponential distribution. Habibullah and Ahsanullah [15] obtained the estimates of the parameters of Pareto type II distribution based on GOS. Also, estimators based on order statistics and record values are obtained as special cases. Jaheen [16] estimated the parameters of the Burr type XII distribution based on GOS and upper record statistics using maximum likelihood (ML), Bayesian and approximate Bayes due to Lindley [20] methods. Mailinowska et al. [22] derived the minimum variance linear unbiased estimators for Burr XII model based on $n$-selected GOS. 
Aboeleneen [3] discussed Bayesian and non-Bayesian estimation methods based on GOS for Weibull distribution. Estimates of the unknown parameters and confidence intervals from progressively type II censoring and record values are obtained. Burkschat [11] derived the best linear unbiased and best equivariant estimators in location and scale families of GOS from generalized Pareto distribution. Abo-Elfotouh and Nassar [4] obtained the estimators of the unknown parameters of the Weibull extension model based on GOS using maximum likelihood and Bayesian methods. Abd-Ellah [2] obtained the estimators of the unknown parameters of the inverse Weibull distribution based on GOS using maximum likelihood and Bayesian methods. Bayes estimates under various loss functions such as the balance squared error and balance LINEX are examined.

Recently, Moghadam, et al. [23] used the maximum likelihood and Bayesian methods to estimate the unknown parameters of the Lomax distribution based on GOS. Safi and Ahmed [24] obtained the estimators of the unknown parameters of the Kumaraswamy distribution based on GOS using maximum likelihood method. Ateya [7] discussed Bayesian and non-Bayesian estimation methods based on right censored GOS for modified Weibull distribution. AbdElfattah, et al. [1] obtained maximum likelihood estimators and confidence intervals of the unknown parameters from exponentiated Weibull Poisson (EWP) distribution based on GOS. In addition, maximum likelihood estimators based on progressively type-II censored data were derived. Kim and Han [18] obtained Bayesian estimators and highest posterior density credible intervals for the scale parameter of Rayleigh distribution based GOS. Also, they derived the Bayesian predictive estimator and the highest posterior density predictive interval for independent future observations.

Mahmoudi and Sepahdar [21] introduced a new compounding distribution with decreasing, increasing, bathtub shaped and unimodel failure rate called as the exponentiated Weibull Poisson distribution. This distribution contains several lifetime sub-models such as: generalized exponentiated Poisson, complementary Weibull Poisson, complementary exponential Poisson, exponential Rayleigh Poisson and Raleigh Poisson distributions.

The probability density function (pdf) of EWP takes the following form

$f(x ; \alpha, \lambda, \theta, \beta)=\frac{\alpha \lambda \theta \beta^{\lambda} x^{\lambda-1}}{\left(e^{\theta}-1\right)} e^{-(\beta x)^{\lambda}}\left(1-e^{-(\beta x)^{\lambda}}\right)^{\alpha-1} e^{\theta\left(1-e^{-(\beta x)^{\lambda}}\right)^{\alpha}} ; x>0, \quad \alpha, \lambda, \theta, \beta>0$

where, $\theta$ and $\beta$ are the scale parameters and $\alpha$ and $\lambda$ are the shape parameters of the distribution.

The corresponding distribution function is given by

$F(x ; \alpha, \lambda, \theta, \beta)=\frac{e^{\theta\left(1-e^{-(\beta x)^{\lambda}}\right)^{\alpha}}-1}{\left(e^{\theta}-1\right)} ; \quad x>0, \quad \alpha, \lambda, \theta, \beta>0$

A wide variety of loss functions have been developed in literature to describe various types of loss structures. The symmetric squared error (SE) loss is one of the useful symmetric loss functions and it is popular due to its relationship to classical least squares theory. The similarity between the two makes SE loss function seem familiar to statisticians. A loss function should represent the consequences of different errors. There are situations where over and under estimation can lead to different consequences. For example, when estimating the average reliable working life of the components of a spaceship or an aircraft, over-estimation is usually more serious than under-estimation. Being symmetric, the SE loss equally penalize over and under estimation of the same magnitude.

Let $u(\phi)$ be a general function of the vector of parameters $\phi=\left(\phi_{1}, \phi_{2}, \ldots, \phi_{r}\right)$. Under the squared error loss function, $L[\tilde{u}(\phi), u(\phi)]=[\tilde{u}(\phi)-u(\phi)]^{2}$, the Bayes estimates of $u(\phi)$ is given by

$\tilde{u}(\phi)=E(\tilde{u}(\phi))=\int \ldots \int u(\phi) \pi(u(\phi) \mid \underline{x}) d \phi_{1} \ldots d \phi_{r}$

The integrals are taken over the $r$-dimensional space (see Koch [19]). The usual estimator of the parameters under the quadratic loss function is the posterior mean.

Linear exponential (LINEX) loss function was introduced by Varian [26] as asymmetric loss function. This function rise approximately exponentially on one side of zero, and approximately linearly on the other side. Under the assumption that the minimal loss occurs at $\tilde{\tilde{u}}(\phi)=u(\phi)$, the LINEX loss function can be expressed as

$L[\Delta)] \propto e^{a \Delta}-a \Delta-1, \quad a \neq 0$,

where, $\Delta=[\tilde{\tilde{u}}(\phi)-u(\phi)], \tilde{\tilde{u}}(\phi)$ is an estimate of $u(\phi)$. The constant parameter $(a)$ determines the shape of the loss function. If the parameter $a>0$ and the error $[\tilde{\tilde{u}}(\phi)-u(\phi)]$ is positive the LINEX loss function is almost exponential and for negative errors almost linear, in these situations over-estimations are more serious problem than underestimation. If $a<0$ under-estimation is more important than over-estimation. For small values of $|a|$, the loss is an almost symmetric and behaves like the SE loss function. Under LINEX loss function, the Bayes estimats of $u(\phi)$ is given by

$\tilde{\tilde{u}}(\phi)=-\frac{1}{a} \ln E\left(e^{-a u(\phi)} \mid \underline{x}\right), \quad a \neq 0$, 
provided that $E\left(e^{-a u(\phi)} \mid \underline{x}\right)$ exists, and is finite. (see Zellner [27]).

Despite the flexibility of the LINEX loss function for the estimation of a location parameter, it appears not to be suitable for the estimation of scale parameter and other quantities. For these reasons, Basu and Ibrahimi [9] proposed the modified LINEX loss function. Calabria and Pulcini [12] presented another alternative to the modified LINEX loss function named general entropy (GE) loss function when it appears to be realistic to express the loss in terms of the ratio between unknown parameters and it's estimators $\left(\frac{\widehat{\widetilde{u}}(\phi)}{u(\phi)}\right)$, and defined it as

$L[\widehat{\tilde{u}}(\phi), u(\phi)] \propto\left(\frac{\widehat{u}(\phi)}{u(\phi)}\right)^{v}-v \log \left(\frac{\widehat{\hat{u}}(\phi)}{u(\phi)}\right)-1$,

whose minimum occurs at $\hat{\tilde{u}}(\phi)=u(\phi)$. This loss function is a generalization of the Entropy loss, where $v=1$ according to Dey et al. [13] and Dey and Liu [14]. When $v>0$, a positive error $[\hat{\tilde{u}}(\phi)>u(\phi)]$ causes serious consequences than a negative error. The Bayes estimates $\hat{\tilde{u}}(\phi)$ of $u(\phi)$ under GE loss is

$\hat{\tilde{u}}(\phi)=\left[E\left([u(\phi)]^{-v}\right)\right]^{-1 / v}$,

provided that $E\left[(u(\phi))^{-v}\right]$ exists and is finite, where $E$ denotes the expected value with respect to the posterior function of $u(\phi)$.

This article is concerned with the Bayesian estimation for the four parameters $(\alpha, \lambda, \theta, \beta)$ of the exponentiated Weibull Poisson based on GOS. This was done under assumption of symmetric (square error) and asymmetric (LINEX and general entropy) loss functions. Numerical study is used to compute the Bayes estimates. The results are specialized to progressive type II censored and the joint density function of ordinary order statistics values.

This article can be organized as follows. Section 2 presents the Bayesian estimators of the unknown parameters for EWP distribution using the symmetric and asymmetric loss functions based on generalized order statistics. The Bayes estimates based on progressive type II censored sample and joint density function of ordinary order statistics are derived in Section 3. In Section 4, numerical computation is developed to illustrate theoretical results. Numerical results are displayed in Section 5. Finally, conclusions are presented in Section 6. Tables are displayed in the appendix.

\section{Bayesian estimators based on GOS}

Suppose that $X(1, n, m, k), X(2, n, m, k), \ldots, X(n, n, m, k)$ are $n$ random sample of GOS drawn from EWP distribution with pdf (2) and define $u(\phi)=(\alpha, \lambda, \theta, \beta)$; where $\phi \equiv(\alpha, \lambda, \theta, \beta)$. Then based on this set of GOS the likelihood function is

$L[u(\phi) ; \underline{x}]=C_{n-1} \alpha^{n} \lambda^{n} \theta^{n} \beta^{n \lambda}\left(e^{\theta}-1\right)^{-\left(n+d+\sum_{i=1}^{n-1} q_{i}\right)} J[u(\phi)]$

where, $J[u(\phi)]=\left[\prod_{i=1}^{n} x_{(i)}^{\lambda-1}\right]\left[\prod_{i=1}^{n}\left(E_{i}\right)^{\alpha-1}\right] \exp \left[\theta \sum_{i=1}^{n}\left(E_{i}\right)^{\alpha}-\sum_{i=1}^{n}\left(\beta x_{(i)}\right)^{\lambda}\right]$ $\sum_{q_{1}=0}^{m_{1}} \ldots \sum_{q_{n-1}=0}^{m_{n-1}} \sum_{d=0}^{k-1}\left(\begin{array}{c}m_{1} \\ q_{1}\end{array}\right) \ldots\left(\begin{array}{c}m_{n-1} \\ q_{n-1}\end{array}\right)\left(\begin{array}{c}k-1 \\ d\end{array}\right)(-1)^{d+\sum_{i=1}^{n-1} q_{i}}\left[e^{\theta\left(E_{i}\right)^{\alpha}}-1\right]^{\sum_{i=1}^{n-1} q_{i}}\left[e^{\theta\left(E_{n}\right)^{\alpha}}-1\right]^{d}$,

$C_{n-1}=k\left(\prod_{j=1}^{n-1} \gamma_{j}\right), \gamma_{j}=k+n-j+\prod_{i=j}^{n-1} m_{i}, E_{i}=\left(1-e^{-\left(\beta x_{(i)}\right)^{\lambda}}\right)$, and $E_{n}=\left(1-e^{-\left(\beta x_{(n)}\right)^{\lambda}}\right)$.

Following Singh, et al. [25] the non-informative type of prior (NIP) for the parameters $\alpha, \lambda, \theta$ and $\beta$ is considered as

$\begin{array}{lll}g_{1}(\alpha) \propto \frac{1}{\alpha} ; & 0<\alpha<\infty, & g_{2}(\lambda) \propto \frac{1}{\lambda} ; \quad 0<\lambda<\infty, \\ g_{3}(\theta) \propto \frac{1}{q_{1}} ; & 0<\theta<q_{1}, q_{1}>0, & g_{4}(\beta) \propto \frac{1}{q_{2}} ; \quad 0<\beta<q_{2}, q_{2}>0 .\end{array}$

Consequently, the joint NIP will be as follows:

$g[u(\phi)] \propto \frac{1}{\alpha \lambda q_{1} q_{2}} ; \alpha, \lambda>0,0<\theta<q_{1}, 0<\beta<q_{2}, q_{1}, q_{2}>0$.

Combining the joint prior density of $u(\phi)=u(\alpha, \lambda, \theta, \beta)$ in (5) and the likelihood function (4) to obtain the joint posterior density of $u(\phi)$ given the data as follows 
$\pi(u(\phi) \mid \underline{x})=\frac{C_{n-1} \alpha^{n-1} \lambda^{n-1} \theta^{n} \beta^{n \lambda}\left(e^{\theta}-1\right)^{-\left(n+d+\sum_{i=1}^{n-1} q_{i}\right)} J[u(\phi)]}{J_{1}} ; \quad \alpha, \lambda>0,0<\theta<q_{1}, 0<\beta<q_{2}, q_{1}, q_{2}>0$.

where, $J_{1}=C_{n-1} \int_{0}^{\infty} \int_{0}^{\infty} \int_{0}^{q_{1}} \int_{0}^{q_{2}} \alpha^{n-1} \lambda^{n-1} \theta^{n} \beta^{n \lambda}\left(e^{\theta}-1\right)^{-\left(n+d+\sum_{i=1}^{n-1} q_{i}\right)} J[u(\phi)] \quad d \beta d \theta d \lambda d \alpha$.

Therefore, based on GOS the Bayes estimates of the unknown parameters $u(\phi)=u(\alpha, \lambda, \theta, \beta)$ under squared error loss function, denoted by $\tilde{u}_{(B E S L)}(\phi)$, can be obtained as posterior mean as follows

$$
\tilde{u}_{(B E S L)}(\phi)=E(u(\phi) \mid \underline{x})=\int_{0}^{\infty} \int_{0}^{\infty} \int_{0}^{q_{1}} \int_{0}^{q_{2}} u(\phi) \pi(u(\phi) \mid \underline{x}) d \beta d \theta d \lambda d \alpha .
$$

Alternatively, under the LINEX loss function the Bayes estimates of $u(\phi)$; denoted by $\tilde{\tilde{u}}_{(B E L L)}(\phi)$; is given by

$$
\tilde{\tilde{u}}_{(B E L L)}(\phi)=-\frac{1}{a} \log \left[E\left(e^{-a u(\phi)} \mid \underline{x}\right)\right]=-\frac{1}{a} \log \left[\int_{0}^{\infty} \int_{0}^{\infty} \int_{0}^{q_{1}} \int_{0}^{q_{2}} e^{-a u(\phi)} \pi(u(\phi) \mid \underline{x}) d \beta d \theta d \lambda d \alpha\right] .
$$

Moreover, the Bayes estimates of $u(\phi)$ under general entropy; denoted by $\hat{\tilde{u}}_{(B E G L)}(\phi)$; can be obtained as follows

$$
\widehat{\tilde{u}}_{(B E G L)}(\phi)=\left(E\left[(u(\phi))^{-v}\right]\right)^{-\frac{1}{v}}=\left[\int_{0}^{\infty} \int_{0}^{\infty} \int_{0}^{q_{1}} \int_{0}^{q_{2}}[u(\phi)]^{-v} \pi(u(\phi) \mid \underline{x}) d \beta d \theta d \lambda d \alpha\right]^{-\frac{1}{v}}
$$

The integral Equations (7) to (9) are very hard to obtain, therefore a numerical procedure is applied to obtain the Bayes estimates of the unknown parameters.

\section{Special cases}

In this section, two special cases of GOS, progressively type II censored and the joint density function of ordinary order statistics will be considered.

\subsection{Bayesian estimators based on progressively type II censored data}

A progressively type II censored sample is observed as follows: $n$ units are placed on a life testing experiment and only $m \leq n$ are completely observed until failure. The censoring occurs progressively in $m$ stages. The $m$ stages are failure times of $m$ completely observed units. At the time of the first failure (the first stage), $R_{1}$ on $(n-1)$ surviving units are randomly withdrawn from the experiment, $R_{2}$ on $\left(n-R_{1}-2\right)$ surviving units are withdrawn at the time of the second failure (the second stage) and so on. Finally, at the time of the $m^{\text {th }}$ failure (the $m^{\text {th }}$ stage), all the remaining $\left(R_{m}=n-m-R_{1}-\cdots-R_{m-1}\right)$ surviving units are withdrawn. In this scheme $\left(R_{1}, R_{2}, \ldots, R_{m}\right)$ is prefixed. The resulting $m$ order failure times, which denote by $X_{i: n: N}^{\left(R_{1}, \ldots, R_{m}\right)}, i=1,2, \ldots, m$ are referred to as progressive type-II right censored order statistics [see Balakrishnan and Aggarwala [8]].

According to Burkschat et al. [10] the progressively type II censored sample $X_{1: n: N}^{\left(R_{1}, \ldots, R_{m}\right)}, \ldots, X_{n: n: N}^{\left(R_{1}, \ldots, R_{m}\right)}$, with censoring scheme $\tilde{R}=\left(R_{1}, R_{2}, \ldots, R_{n}\right)$, and $R_{i} \in N, 1 \leq i \leq n$ is special case of the generalized order statistics with the parameter $m_{i}=R_{i}, i=1,2, \ldots, n-1$ and $k=\gamma_{n}=R_{n}+1$. Therefore, the likelihood function (4), is reduced to likelihood function of progressive type II censored data as follows

$L_{1}[u(\phi) ; \underline{x}]=C_{n-1} \alpha^{n} \lambda^{n} \theta^{n} \beta^{n \lambda}\left(e^{\theta}-1\right)^{-\left(n+\sum_{i=1}^{n} R_{i}\right)} Q[u(\phi)]$,

where, $Q[u(\phi)]=\left[\prod_{i=1}^{n} x_{(i)}^{\lambda-1}\right]\left[\prod_{i=1}^{n}\left(E_{i}\right)^{\alpha-1}\right] \exp \left[\theta \sum_{i=1}^{n}\left(E_{i}\right)^{\alpha}-\sum_{i=1}^{n}\left(\beta x_{(i)}\right)^{\lambda}\right]$

$$
\sum_{q_{1}=0}^{R_{1}} \cdots \sum_{q_{n}=0}^{R_{n}}\left(\begin{array}{c}
R_{1} \\
q_{1}
\end{array}\right) \ldots\left(\begin{array}{c}
R_{n} \\
q_{n}
\end{array}\right)(-1)^{\sum_{i=1}^{n} R_{i}}\left[e^{\theta\left(E_{i}\right)^{\alpha}}-1\right]^{\sum_{i=1}^{n} R_{i}}
$$

$\left.C_{n-1}=k\left(\prod_{j=1}^{n-1} \gamma_{j}\right), \gamma_{j}=k+n-j+\prod_{i=j}^{n-1} m_{i}, E_{i}=\left(1-e^{-\left(\beta x_{(i)}\right.}\right)^{\lambda}\right)$, and $E_{n}=\left(1-e^{-\left(\beta x_{(n)}\right)^{\lambda}}\right)$.

To obtain the joint posterior density of $u(\phi)$, combining the joint prior density (5) and the likelihood function (10) as

$\pi_{1}(u(\phi) \mid \underline{x})=\frac{c_{n-1} \alpha^{n-1} \lambda^{n-1} \theta^{n} \beta^{n \lambda}\left(e^{\theta}-1\right)^{-\left(n+\sum_{i=1}^{n-1} q_{i}\right)} Q[u(\phi)]}{J_{2}} ; \quad \alpha, \lambda>0,0<\theta<q_{1}, 0<\beta<q_{2}, q_{1}, q_{2}>0$ 
where, $J_{2}=C_{n-1} \int_{0}^{\infty} \int_{0}^{\infty} \int_{0}^{q_{1}} \int_{0}^{q_{2}} \alpha^{n-1} \lambda^{n-1} \theta^{n} \beta^{n \lambda}\left(e^{\theta}-1\right)^{-\left(n+\sum_{i=1}^{n-1} q_{i}\right)} Q[u(\phi)] \quad d \beta d \theta d \lambda d \alpha$.

Therefore, the posterior mean of the unknown parameters $u(\phi)=u(\alpha, \lambda, \theta, \beta)$; denoted by $\tilde{u}_{1(B E S L)}(\phi)$; based on progressive type II censored data is obtained as follows

$\tilde{u}_{1(B E S L)}(\phi)=E(u(\phi) \mid \underline{x})=\int_{0}^{\infty} \int_{0}^{\infty} \int_{0}^{q_{1}} \int_{0}^{q_{2}} u(\phi) \pi_{1}(u(\phi) \mid \underline{x}) d \beta d \theta d \lambda d \alpha$.

Based on, the LINEX loss function the Bayesian estimators of $u(\phi)$; denoted by $\tilde{\tilde{u}}_{1(B E L L)}(\phi)$; can be obtained as

$\tilde{\tilde{u}}_{1(B E L L)}(\phi)=-\frac{1}{a} \log \left[E\left(e^{-a u(\phi)} \mid \underline{x}\right)\right]=-\frac{1}{a} \log \left[\int_{0}^{\infty} \int_{0}^{\infty} \int_{0}^{q_{1}} \int_{0}^{q_{2}} e^{-a u(\phi)} \pi_{1}(u(\phi) \mid \underline{x}) d \beta d \theta d \lambda d \alpha\right]$.

Furthermore, the Bayesian estimators of $u(\phi)$ under general entropy; denoted by $\hat{\tilde{u}}_{1(B E G L)}(\phi)$; is given by

$\hat{\tilde{u}}_{1(B E G L)}(\phi)=\left(E\left[(u(\phi))^{-v}\right]\right)^{-\frac{1}{v}}=\left[\int_{0}^{\infty} \int_{0}^{\infty} \int_{0}^{q_{1}} \int_{0}^{q_{2}}(u(\phi))^{-v} \pi_{1}(u(\phi) \mid \underline{x}) d \beta d \theta d \lambda d \alpha\right]^{-\frac{1}{v}}$.

As mentioned earlier, the integrals involved in (12), (13) and (14) are not solvable analytically and, therefore, a numerical technique and computer facilities are needed to evaluate the Bayes estimate of the unknown parameters.

\subsection{Bayesian estimators based on the joint density function of ordinary order statistics}

The joint density function of ordinary order statistics is special case of generalized order statistics, by setting $m_{1}=m_{2}=\cdots=m_{n-1}=0$ and $k=1$ in Equation (4), then the likelihood function of the joint density function of ordinary order statistics; denoted by $L_{2}[u(\phi) ; \underline{x}]$; takes the following form

$L_{2}[u(\phi) ; \underline{x}]=C_{n-1} \alpha^{n} \lambda^{n} \theta^{n} \beta^{n \lambda}\left(e^{\theta}-1\right)^{-n} A[u(\phi)]$

where, $C_{n-1}=k\left(\prod_{j=1}^{n-1} \gamma_{j}\right)$ and $A[u(\phi)]=\left[\prod_{i=1}^{n} x_{(i)}^{\lambda-1}\right]\left[\prod_{i=1}^{n}\left(E_{i}\right)^{\alpha-1}\right] \exp \left[\theta \sum_{i=1}^{n}\left(E_{i}\right)^{\alpha}-\sum_{i=1}^{n}\left(\beta x_{(i)}\right)^{\lambda}\right]$.

Combining the joint prior density (5) and the likelihood function (15) to obtain the joint posterior density of $u(\phi)$ as follows;

$\pi_{2}(u(\phi) \mid \underline{x})=\frac{C_{n-1} \alpha^{n-1} \lambda^{n-1} \theta^{n} \beta^{n \lambda}\left(e^{\theta}-1\right)^{-n} A[u(\phi)]}{J_{3}} ; \quad \alpha, \lambda>0,0<\theta<q_{1}, \quad 0<\beta<q_{2}, q_{1}, q_{2}>0$,

where, $J_{3}=C_{n-1} \int_{0}^{\infty} \int_{0}^{\infty} \int_{0}^{q_{1}} \int_{0}^{q_{2}} \alpha^{n-1} \lambda^{n-1} \theta^{n} \beta^{n \lambda}\left(e^{\theta}-1\right)^{-n} A[u(\phi)] d \beta d \theta d \lambda d \alpha$.

Therefore, the Bayes estimates of the unknown parameters $u(\phi)=u(\alpha, \lambda, \theta, \beta)$ based on the joint density function of ordinary order statistics under square error loss function, LINEX and general entropy; denoted by $\tilde{u}_{2(B E S L)}(\phi)$, $\tilde{\tilde{u}}_{2(B E L L)}(\phi)$ and $\widehat{\widetilde{u}}_{2(B E G L)}(\phi)$ respectively; can be calculated through the following equations as follows

$\tilde{u}_{2(B E S L)}(\phi)=E(u(\phi) \mid \underline{x})=\int_{0}^{\infty} \int_{0}^{\infty} \int_{0}^{q_{1}} \int_{0}^{q_{2}} u(\phi) \pi_{2}(u(\phi) \mid \underline{x}) d \beta d \theta d \lambda d \alpha$,

$\tilde{\tilde{u}}_{2(B E L L)}(\phi)=-\frac{1}{a} \log \left[E\left(e^{-a u(\phi)} \mid \underline{x}\right)\right]=-\frac{1}{a} \log \left[\int_{0}^{\infty} \int_{0}^{\infty} \int_{0}^{q_{1}} \int_{0}^{q_{2}} e^{-a u(\phi)} \pi_{2}(u(\phi) \mid \underline{x}) d \beta d \theta d \lambda d \alpha\right]$,

$\hat{\tilde{u}}_{2(B E G L)}(\phi)=\left(E\left[(u(\phi))^{-v}\right]\right)^{-\frac{1}{v}}=\left[\int_{0}^{\infty} \int_{0}^{\infty} \int_{0}^{q_{1}} \int_{0}^{q_{2}}(u(\phi))^{-v} \pi_{2}(u(\phi) \mid \underline{x}) d \beta d \theta d \lambda d \alpha\right]^{-\frac{1}{v}}$.

The integrals involved in (17), (18) and (19) can't be solved analytically, so it's necessary to apply a numerical technique and computer facilities to evaluate the Bayes estimate of unknown parameters.

\section{Numerical illustration}

In any estimation problems, it is required to study the properties of the derived estimators. The derived expressions for the estimators are too complicated to study analytically. Consequently, a simulation study will be set up for illustrating the theoretical results via MathCAD 14. The performance of the resulting estimators of the unknown 
parameters has been considered in terms of their mean square error (MSE), and estimated risk (ER). The simulation procedures will be described below:

Step 1: Following Abo-Elfotouh and Nassar [4] 100 random samples of sizes 20, 30, 40, 100, 150 and 200 are generated from EWP distribution under GOS and the joint density function of ordinary order statistics. Also, 100 random samples of sizes 30, 50, 100, 150 and 200 are generated from EWP distribution under progressive type II censoring data.

Step 2: Selected two set of parameters values as case $\mathrm{I} \equiv(\alpha=0.25, \lambda=0.8, \theta=0.5, \beta=1)$, case II $\equiv(\alpha=$ $0.75, \lambda=1.5, \theta=0.5, \beta=1)$ for $(k=1.025, m=0.03)$.

Step 3: Numerical technique is applied for solving Equations (7), (8) and (9) for the unknown parameters $u(\phi)$ to obtain the Bayesian estimators under squared error, LINEX and general entropy loss functions based on GOS.

Step 4: The integral Equations (12) to (14) are calculated numerically to obtain the Bayes estimates; $\tilde{u}_{1(B E S L)}(\phi)$, $\tilde{\tilde{u}}_{1(B E L L)}(\phi)$ and $\widehat{\tilde{u}}_{1(B E G L)}(\phi)$, under symmetric and asymmetric loss functions based on progressive type II censored.

Step 5: Based on the joint density function of ordinary order statistics the Bayes estimates, $\tilde{u}_{2(B E S L)}(\phi), \tilde{\tilde{u}}_{2(B E L L)}(\phi)$ and $\widehat{\tilde{u}}_{2(B E G L)}(\phi)$ are calculated by numerically solving Equations (17), (18) and (19).

Step 6: MSE and ER of all estimators are tabulated based on GOS, progressive type II censored and joint density function of ordinary order statistics.

\section{Simulation results}

Simulation results are summarized in Tables 1 to 6 . Tables 1 and 2 give the MSE and ER of the Bayesian estimators based on GOS. Tables 3 and 4 give the MSE, and ER of the Bayesian estimators under joint density function of ordinary order statistics. Also, Tables 5 to 6 give the MSE and ER of the Bayesian estimators under progressive type II censored data. Based on the three techniques of estimation, MSEs and ERs are calculated for the selected set of parameters and different sample sizes.

From these tables, the following observations can be made on the performance of estimated parameters of EWP lifetime distribution based on GOS, the joint density function of ordinary order statistics and progressive type II censored samples:

1) MSE of the Bayes estimates for the first set of parameters $(\alpha=0.25, \lambda=0.8, \theta=0.5, \beta=1)$ is smaller than MSE of the Bayes estimates for the second set of parameters $(\alpha=0.75, \lambda=1.5, \theta=0.5, \beta=1)$ for all sample sizes under GOS. While ER of the second set of parameters is smaller than ER of the first set of parameters for all sample size under GOS (see Tables 1 and 2).

2) MSE of the Bayes estimates for the first set of parameters $(\alpha=0.25, \lambda=0.8, \theta=0.5, \beta=1)$ for $(k=1, m=$ $O$ is smaller than MSE of the of the Bayes estimates for the second set of parameters based on joint density function of ordinary order statistics. Also, ER of the second set of parameters is smaller than ER of the first set of parameters based on joint density function of ordinary order statistics (see Tables 3 and 4).

3) MSE of the Bayes estimates for the first set of parameters $(\alpha=0.25, \lambda=0.8, \theta=0.5, \beta=1)$ for $(k=1, m=$ $O$ is smaller than MSE of the of the Bayes estimates for the second set of parameters based progressive type II censored data. While, ER of the second set of parameters is smaller than ER of the first set of parameters based on progressive type II censored data (see Tables 5 and 6).

4) MSEs of asymmetric (LINEX, general entropy) Bayes estimates are over-estimates for $(a<0, v<0)$, and when $(a>0, v>0)$ the MSEs of Bayes estimates are under-estimates. As anticipated, the MSEs of asymmetric Bayes estimates are the same as the MSEs of Bayes estimates relative to SE loss function (for a close to 0 , and $v=-1$ ). This is one of the useful properties of working with the asymmetric loss functions.

5) Bayes estimates under the GE loss function have the smallest estimated MSEs as compared with the Bayes estimates under the LINEX and SE loss functions based on generalized order statistics, the joint density function of ordinary order statistics and progressive type II censoring.

6) The MSEs of both estimated parameters $\theta$ and $\beta$ is smaller than the MSEs of both estimated parameters $\alpha$ and $\lambda$ in almost all the cases.

7) Based on progressively type II censoring, the ER for the estimates of the unknown parameters under GE loss function has smaller values than the corresponding ER under GE loss function based on GOS and the joint density function of ordinary order statistics.

8) When the effective sample proportion $m / n$ Increases, the MSE of different Bayesian estimators is reduced, also the censoring scheme of the progressively type II censored data is most efficient for all choices. It usually provides the smallest MSE for all estimators.

9) The MSE and ER of the Bayesian estimators are computed over different combination of the censored scheme as shown in Tables 5 to 6 .

10) For all methods, clearly MSEs and ERs decrease as sample sizes increase for all estimates.

11) For fixed values of $\theta$ and $\beta$ as the values of parameters $\alpha$ and $\lambda$ Increase, the MSEs for estimators increase based on GOS, joint density function of ordinary order statistics and progressive type II censoring. 


\section{Appendix}

Table 1: Mean square error and estimated risk of the Bayes estimates under SE, LINEX and GE loss functions based on GOS for $(\alpha=0.25, \lambda=0.8, \theta=0.5, \beta=1)$

\begin{tabular}{|c|c|c|c|c|c|c|c|c|c|c|c|}
\hline \multirow{3}{*}{$n$} & \multirow{3}{*}{ Parameters } & \multirow{2}{*}{\multicolumn{2}{|c|}{ SE Loss Function }} & \multicolumn{4}{|c|}{ LINEX Loss Function } & \multicolumn{4}{|c|}{ GE Loss Function } \\
\hline & & & & \multicolumn{2}{|c|}{$a=-4$} & \multicolumn{2}{|c|}{$a=4$} & \multicolumn{2}{|c|}{$v=-4$} & \multicolumn{2}{|c|}{$v=4$} \\
\hline & & MSE & ER & MSE & ER & MSE & ER & MSE & ER & MSE & ER \\
\hline \multirow{4}{*}{20} & $\alpha$ & 0.413 & 0.130 & 0.431 & 0.386 & 0.101 & 0.086 & 0.361 & 0.067 & 0.054 & 0.016 \\
\hline & $\lambda$ & 0.151 & 0.034 & 0.531 & $5.575^{*}$ & 0.135 & 0.085 & 0.254 & $0.936 *$ & 0.130 & 0.014 \\
\hline & $\theta$ & 0.145 & 0.065 & 0.438 & $8.040^{*}$ & 0.123 & 0.016 & 0.011 & $5.729 *$ & 0.010 & $4.766^{*}$ \\
\hline & $\beta$ & 0.149 & $0.189 *$ & 0.169 & $7.312 *$ & 0.017 & 0.011 & 0.160 & $1.581 *$ & 0.016 & $0.097 *$ \\
\hline \multirow{4}{*}{30} & $\alpha$ & 0.313 & 0.054 & 0.356 & 0.350 & 0.076 & 0.054 & 0.298 & 0.049 & 0.050 & $9.672 *$ \\
\hline & $\lambda$ & 0.125 & 0.014 & 0.491 & $4.638 *$ & 0.085 & $9.172 *$ & 0.239 & $0.554 *$ & 0.073 & $3.568 *$ \\
\hline & $\theta$ & 0.024 & 0.011 & 0.376 & $1.514^{*}$ & 0.005 & $7.086^{*}$ & $5.233^{*}$ & $2.565^{*}$ & $4.257^{*}$ & $1.733 *$ \\
\hline & $\beta$ & 0.146 & $0.387 *$ & 0.154 & $1.262 *$ & 0.016 & $6.036^{*}$ & 0.151 & $0.772 *$ & 0.012 & $0.057 *$ \\
\hline \multirow{4}{*}{40} & $\alpha$ & 0.279 & 0.053 & 0.333 & 0.329 & 0.068 & 0.048 & 0.289 & 0.039 & 0.034 & $8.622 *$ \\
\hline & $\lambda$ & 0.119 & 0.011 & 0.465 & $0.895^{*}$ & 0.075 & $7.531 *$ & 0.238 & $0.258 *$ & 0.061 & $3.208 *$ \\
\hline & $\theta$ & 0.023 & 0.035 & 0.374 & $1.605^{*}$ & $8.247 *$ & $2.820^{*}$ & $5.030 *$ & $2.315^{*}$ & $3.951 *$ & $0.464 *$ \\
\hline & $\beta$ & 0.144 & $0.278 *$ & 0.154 & $1.248 *$ & 0.014 & $2.342 *$ & 0.147 & $0.251 *$ & 0.012 & $0.047 *$ \\
\hline \multirow{4}{*}{100} & $\alpha$ & 0.272 & 0.046 & 0.225 & 0.215 & 0.057 & 0.048 & 0.042 & 0.012 & 0.033 & $8.008^{*}$ \\
\hline & $\lambda$ & 0.106 & $7.549 *$ & 0.464 & $0.747 *$ & 0.066 & $2.946^{*}$ & 0.236 & $0.294 *$ & 0.045 & $3.033^{*}$ \\
\hline & $\theta$ & $4.307 *$ & $1.548 *$ & 0.346 & $0.260 *$ & $6.815^{*}$ & $1.514 *$ & $3.756^{*}$ & $1.506 *$ & $0.9395^{*}$ & $0.326^{*}$ \\
\hline & $\beta$ & 0.144 & $0.229 *$ & 0.152 & $0.261^{*}$ & 0.013 & $1.262 *$ & 0.132 & $0.025^{*}$ & 0.011 & $0.039 *$ \\
\hline \multirow{4}{*}{150} & $\alpha$ & 0.168 & 0.037 & 0.219 & 0.192 & 0.049 & 0.046 & 0.032 & $9.266^{*}$ & 0.020 & $4.537 *$ \\
\hline & $\lambda$ & 0.073 & $3.208 *$ & 0.438 & $0.135^{*}$ & 0.040 & $1.946^{*}$ & 0.215 & $0.106 *$ & 0.026 & $2.547 *$ \\
\hline & $\theta$ & $1.266^{*}$ & $1.266^{*}$ & 0.324 & $0.161^{*}$ & $5.705 *$ & $1.042 *$ & $1.028 *$ & $0.365^{*}$ & $0.5134 *$ & $0.189 *$ \\
\hline & $\beta$ & 0.138 & $0.041 *$ & 0.152 & $0.162 *$ & 0.011 & $1.248^{*}$ & 0.125 & $0.011 *$ & 0.009 & 0.001 * \\
\hline \multirow{4}{*}{200} & $\alpha$ & 0.136 & $9.065 *$ & 0.174 & 0.085 & 0.027 & 0.013 & 0.169 & $6.631 *$ & 0.016 & $2.160 *$ \\
\hline & $\lambda$ & 0.049 & $1.427 *$ & 0.361 & $0.069 *$ & 0.032 & $0.837 *$ & 0.340 & $0.057 *$ & 0.020 & $1.795 *$ \\
\hline & $\theta$ & $0.907 *$ & $0.839 *$ & 0.303 & $0.095^{*}$ & $4.362 *$ & $0.873^{*}$ & 0.273 & $0.139 *$ & $3.692 *$ & $0.074 *$ \\
\hline & $\beta$ & 0.079 & $0.009 *$ & 0.135 & $0.127 *$ & 0.005 & $0.973 *$ & 0.129 & $0.005 *$ & $3.941 *$ & $0.001 *$ \\
\hline
\end{tabular}

Table 2: Mean square error and estimated risk of the Bayes estimates under SE, LINEX and GE loss functions based on GOS for $(\alpha=0.75, \lambda=1.5, \theta=0.5, \beta=1)$

\begin{tabular}{|c|c|c|c|c|c|c|c|c|c|c|c|}
\hline \multirow{3}{*}{$n$} & \multirow{3}{*}{ Parameters } & \multirow{2}{*}{\multicolumn{2}{|c|}{ SE Loss Function }} & \multicolumn{4}{|c|}{ LINEX Loss Function } & \multicolumn{4}{|c|}{ GE Loss Function } \\
\hline & & & & & -4 & & & $v=$ & -4 & & 4 \\
\hline & & MSE & ER & MSE & ER & MSE & ER & MSE & ER & MSE & ER \\
\hline \multirow{4}{*}{20} & $\alpha$ & 1.795 & 0.043 & 2.149 & 0.072 & 1.670 & 0.063 & 1.955 & $6.938^{*}$ & 0.843 & 0.021 \\
\hline & $\lambda$ & 1.013 & 0.010 & 1.982 & $0.652 *$ & 1.008 & $0.413^{*}$ & 1.177 & $0.805^{*}$ & 0.984 & $0.236^{*}$ \\
\hline & $\theta$ & $8.395 *$ & $0.310 *$ & 0.089 & $0.792 *$ & $4.218 *$ & $0.185^{*}$ & 0.630 & $8.495 *$ & $4.197 *$ & $2.902 *$ \\
\hline & $\beta$ & $2.282 *$ & $0.049 *$ & 0.516 & $1.373 *$ & $2.028 *$ & $1.953 *$ & 0.097 & $0.009 *$ & $2.015 *$ & $0.035 *$ \\
\hline \multirow{4}{*}{30} & $\alpha$ & 0.828 & 0.019 & 1.111 & 0.025 & 0.786 & 0.038 & 1.782 & $5.335 *$ & 0.236 & 0.015 \\
\hline & $\lambda$ & 0.750 & $3.837 *$ & 1.877 & $0.175 *$ & 0.705 & $0.132 *$ & 1.020 & $0.451 *$ & 0.668 & $0.209 *$ \\
\hline & $\theta$ & $6.572 *$ & $0.138 *$ & 0.030 & $0.719 *$ & $1.697 *$ & $0.132 *$ & 0.529 & $3.308 *$ & $1.534 *$ & $1.484 *$ \\
\hline & $\beta$ & $1.872 *$ & $0.030 *$ & 0.426 & $0.387 *$ & $1.824 *$ & $0.317 *$ & 0.095 & $0.004 *$ & $1.621 *$ & $0.017 *$ \\
\hline \multirow{4}{*}{40} & $\alpha$ & 0.798 & $5.234 *$ & 0.906 & $7.883 *$ & 0.522 & 0.036 & 1.538 & $5.685 *$ & 0.128 & 0.012 \\
\hline & $\lambda$ & 0.719 & $2.558 *$ & 1.845 & $0.157 *$ & 0.625 & $0.103 *$ & 0.918 & $0.263^{*}$ & 0.596 & $0.202 *$ \\
\hline & $\theta$ & $4.699 *$ & $0.278^{*}$ & 0.020 & $0.415^{*}$ & $1.211 *$ & $0.124 *$ & 0.437 & $2.171 *$ & $1.193 *$ & $1.184 *$ \\
\hline & $\beta$ & $1.434 *$ & $0.012 *$ & 0.420 & $0.317 *$ & $1.220 *$ & $0.247 *$ & 0.093 & $0.003 *$ & $1.219 *$ & $0.006^{*}$ \\
\hline \multirow{4}{*}{100} & $\alpha$ & 0.463 & $3.166^{*}$ & 0.676 & $8.961 *$ & 0.322 & 0.019 & 1.312 & $4.842 *$ & 0.108 & 0.011 \\
\hline & $\lambda$ & 0.623 & $1.804 *$ & 1.807 & $0.117 *$ & 0.568 & $0.078 *$ & 0.896 & $0.242 *$ & 0.516 & $0.186^{*}$ \\
\hline & $\theta$ & $4.568 *$ & $0.124 *$ & 0.016 & $0.223 *$ & $0.540 *$ & $0.079 *$ & 0.352 & $1.569 *$ & $0.498 *$ & $0.582 *$ \\
\hline & $\beta$ & $1.033 *$ & $0.011 *$ & 0.390 & $0.247 *$ & $1.015 *$ & $0.129 *$ & 0.091 & $0.002 *$ & $0.965 *$ & $0.006 *$ \\
\hline \multirow{4}{*}{150} & $\alpha$ & 0.241 & $1.781 *$ & 0.350 & $8.861 *$ & 0.091 & 0.017 & 1.158 & $3.124 *$ & 0.079 & 0.010 \\
\hline & $\lambda$ & 0.367 & $0.818^{*}$ & 1.783 & $0.075^{*}$ & 0.324 & $0.062 *$ & 0.687 & $0.232 *$ & 0.305 & $0.149 *$ \\
\hline & $\theta$ & $2.297 *$ & $0.079 *$ & 0.012 & $0.134 *$ & $0.239 *$ & $0.065^{*}$ & 0.307 & $1.123^{*}$ & $0.230^{*}$ & $0.375^{*}$ \\
\hline & $\beta$ & $0.642 *$ & $0.008 *$ & 0.361 & $0.129 *$ & $0.499 *$ & $0.099 *$ & 0.089 & $0.002 *$ & $0.483^{*}$ & $0.005^{*}$ \\
\hline \multirow{4}{*}{200} & $\alpha$ & 0.186 & $0.946 *$ & 0.291 & $6.354 *$ & 0.133 & 0.006 & 0.282 & $2.451 *$ & 0.119 & 0.007 \\
\hline & $\lambda$ & 0.291 & $0.591 *$ & 1.505 & $0.013 *$ & 0.167 & $0.024 *$ & 1.437 & $0.201 *$ & 0.138 & $0.110^{*}$ \\
\hline & $\theta$ & $1.749 *$ & $0.033^{*}$ & 0.010 & $0.064 *$ & $0.765^{*}$ & $0.041 *$ & $5.739 *$ & $1.033 *$ & $0.439 *$ & $0.175^{*}$ \\
\hline & $\beta$ & $0.602 *$ & $0.006 *$ & 0.313 & $0.044 *$ & $0.385 *$ & $0.079 *$ & 0.286 & $0.001 *$ & $0.276^{*}$ & $0.003 *$ \\
\hline
\end{tabular}

\footnotetext{
* Indicate that the value multiply $10^{-3}$
} 
Table 3: Mean square error and estimated risk of the Bayes estimates under SE, LINEX and GE loss functions based on joint density function of ordinary order statistics for $(\alpha=0.25, \lambda=0.8, \theta=0.5, \beta=1)$

\begin{tabular}{|c|c|c|c|c|c|c|c|c|c|c|c|}
\hline \multirow{3}{*}{$n$} & \multirow{3}{*}{ parameters } & \multirow{2}{*}{\multicolumn{2}{|c|}{ SE Loss Function }} & \multicolumn{4}{|c|}{ LINEX Loss Function } & \multicolumn{4}{|c|}{ GE Loss Function } \\
\hline & & & & & -4 & & & & -4 & & \\
\hline & & MSE & ER & MSE & ER & MSE & ER & MSE & ER & MSE & ER \\
\hline \multirow{4}{*}{20} & $\alpha$ & 0.371 & 0.116 & 0.604 & 0.360 & 0.185 & 0.080 & 0.587 & 0.078 & 0.147 & 0.014 \\
\hline & $\lambda$ & 0.172 & 0.034 & 0.390 & $3.740 *$ & 0.156 & $0.885^{*}$ & 0.353 & $2.248 *$ & 0.145 & $5.863 *$ \\
\hline & $\theta$ & 0.117 & 0.059 & 0.431 & $6.326^{*}$ & 0.101 & $7.073 *$ & 0.392 & 0.095 & 0.044 & $4.839 *$ \\
\hline & $\beta$ & 0.148 & $0.399 *$ & 0.265 & $5.818^{*}$ & 0.119 & 0.011 & 0.232 & $1.553 *$ & 0.071 & $0.099 *$ \\
\hline \multirow{4}{*}{30} & $\alpha$ & 0.300 & 0.077 & 0.458 & 0.316 & 0.074 & 0.066 & 0.428 & 0.069 & 0.036 & $8.201 *$ \\
\hline & $\lambda$ & 0.120 & 0.013 & 0.351 & $2.105^{*}$ & 0.105 & $0.209 *$ & 0.249 & $0.565^{*}$ & 0.100 & $3.527 *$ \\
\hline & $\theta$ & 0.042 & 0.024 & 0.384 & $2.913^{*}$ & 0.025 & $5.687 *$ & 0.256 & $9.931 *$ & $6.684 *$ & $3.636^{*}$ \\
\hline & $\beta$ & 0.147 & $0.366^{*}$ & 0.247 & $2.744 *$ & 0.015 & $5.818^{*}$ & 0.207 & $0.849 *$ & $9.012 *$ & $0.046^{*}$ \\
\hline \multirow{4}{*}{40} & $\alpha$ & 0.295 & 0.067 & 0.416 & 0.266 & 0.074 & 0.060 & 0.381 & 0.053 & 0.030 & 7.618* \\
\hline & $\lambda$ & 0.120 & $7.054 *$ & 0.356 & $1.405^{*}$ & 0.102 & $0.138 *$ & 0.247 & $0.511 *$ & 0.070 & $2.617 *$ \\
\hline & $\theta$ & 0.019 & $9.768 *$ & 0.365 & $1.341^{*}$ & 0.014 & $3.212 *$ & 0.243 & $8.693 *$ & $3.232 *$ & $1.529 *$ \\
\hline & $\beta$ & 0.146 & $0.208 *$ & 0.210 & $1.141 *$ & 0.013 & $3.237^{*}$ & 0.194 & $0.754 *$ & 0.011 & $0.035^{*}$ \\
\hline \multirow{4}{*}{100} & $\alpha$ & 0.256 & 0.065 & 0.390 & 0.240 & 0.064 & 0.058 & 0.372 & 0.040 & 0.025 & $7.406^{*}$ \\
\hline & $\lambda$ & 0.109 & $5.327 *$ & 0.342 & $1.351 *$ & 0.100 & $0.132 *$ & 0.244 & $0.369 *$ & 0.073 & $1.429 *$ \\
\hline & $\theta$ & 0.012 & $6.848^{*}$ & 0.355 & $1.062 *$ & $8.225^{*}$ & 0.012 & 0.230 & $7.559 *$ & $7.326 *$ & $1.283^{*}$ \\
\hline & $\beta$ & 0.150 & $0.159 *$ & 0.187 & $0.848^{*}$ & 0.013 & $2.744^{*}$ & 0.179 & $0.561 *$ & $9.715^{*}$ & $0.023 *$ \\
\hline \multirow{4}{*}{150} & $\alpha$ & 0.109 & 0.053 & 0.299 & 0.168 & 0.030 & 0.035 & 0.134 & 0.027 & 0.020 & $6.409 *$ \\
\hline & $\lambda$ & 0.104 & $4.562 *$ & 0.330 & $0.886^{*}$ & 0.055 & $0.132 *$ & 0.240 & $0.275^{*}$ & 9.093* & $1.328 *$ \\
\hline & $\theta$ & $7.709 *$ & $5.347 *$ & 0.343 & $0.892 *$ & $6.218 *$ & $6.705^{*}$ & 0.177 & $6.185^{*}$ & $5.586 *$ & $1.115^{*}$ \\
\hline & $\beta$ & 0.142 & $0.139 *$ & 0.181 & $0.684 *$ & 0.011 & $2.322 *$ & 0.175 & $0.474 *$ & $8.594 *$ & $0.019 *$ \\
\hline \multirow{4}{*}{200} & $\alpha$ & 0.076 & 0.014 & 0.252 & 0.112 & 0.060 & 0.028 & 0.127 & 0.013 & $7.233 *$ & $3.450 *$ \\
\hline & $\lambda$ & 0.058 & $3.288^{*}$ & 0.291 & $0.643^{*}$ & 0.042 & $0.111^{*}$ & 0.201 & $0.135^{*}$ & $6.093 *$ & $0.557 *$ \\
\hline & $\theta$ & $7.132 *$ & $5.015^{*}$ & 0.301 & $0.743 *$ & $5.874 *$ & $1.341 *$ & 0.170 & $4.811^{*}$ & $4.551 *$ & $0.683 *$ \\
\hline & $\beta$ & 0.096 & $0.104 *$ & 0.087 & $0.438 *$ & $8.632 *$ & $1.141 *$ & 0.137 & $0.386^{*}$ & $6.565 *$ & $0.007 *$ \\
\hline
\end{tabular}

* Indicate that the value multiply $10^{-3}$

Table 4: Mean square error and estimated risk of the Bayes estimates under SE, LINEX and GE loss functions based on joint density function of ordinary order statistics for $(\alpha=0.75, \lambda=1.5, \theta=0.5, \beta=1)$

\begin{tabular}{|c|c|c|c|c|c|c|c|c|c|c|c|}
\hline \multirow{3}{*}{$n$} & \multirow{3}{*}{ Parameters } & \multirow{2}{*}{\multicolumn{2}{|c|}{ SE Loss Function }} & \multicolumn{4}{|c|}{ LINEX Loss Function } & \multicolumn{4}{|c|}{ GE Loss Function } \\
\hline & & & & & -4 & & $=4$ & $v=$ & -4 & & \\
\hline & & MSE & ER & MSE & ER & MSE & ER & MSE & ER & MSE & ER \\
\hline \multirow{4}{*}{20} & $\alpha$ & 1.763 & 0.072 & 2.287 & 0.043 & 1.633 & 0.028 & 2.323 & 0.029 & 1.154 & 0.015 \\
\hline & $\lambda$ & 1.626 & $0.535 *$ & 1.956 & $0.354 *$ & 1.208 & 0.016 & 1.951 & $1.314 *$ & 1.207 & $0.805^{*}$ \\
\hline & $\theta$ & 0.022 & 0.020 & 0.417 & $1.641 *$ & 0.134 & $3.194 *$ & 0.054 & $0.842 *$ & $4.218 *$ & $0.502 *$ \\
\hline & $\beta$ & $2.414 *$ & $0.009 *$ & 0.426 & $0.387 *$ & 0.158 & $0.625 *$ & 0.203 & $0.035 *$ & 0.048 & $0.009 *$ \\
\hline \multirow{4}{*}{30} & $\alpha$ & 0.988 & 0.014 & 1.795 & 0.019 & 0.931 & 0.017 & 1.216 & 0.010 & 0.894 & 0.011 \\
\hline & $\lambda$ & 1.564 & $0.322 *$ & 1.795 & $0.251 *$ & 1.013 & $1.804 *$ & 1.696 & $0.186^{*}$ & 1.003 & $0.203^{*}$ \\
\hline & $\theta$ & 0.016 & 0.012 & 0.365 & $0.872 *$ & 0.098 & $1.699 *$ & 0.027 & $0.569 *$ & $2.197^{*}$ & $0.278 *$ \\
\hline & $\beta$ & $2.047 *$ & $0.004 *$ & 0.390 & $0.247 *$ & 0.130 & $0.513 *$ & 0.195 & $0.006 *$ & 0.048 & $0.008 *$ \\
\hline \multirow{4}{*}{40} & $\alpha$ & 0.822 & 0.013 & 1.607 & 0.017 & 0.714 & 0.016 & 1.120 & $8.910 *$ & 0.697 & $8.177 *$ \\
\hline & $\lambda$ & 1.558 & $0.217 *$ & 1.736 & $0.175^{*}$ & 0.896 & $0.818 *$ & 1.657 & $0.181 *$ & 0.863 & $0.186^{*}$ \\
\hline & $\theta$ & 0.012 & $6.721 *$ & 0.315 & $0.721 *$ & 0.096 & $1.484 *$ & 0.025 & $0.520 *$ & $1.697 *$ & $0.138 *$ \\
\hline & $\beta$ & $2.024 *$ & $0.003 *$ & 0.361 & $0.202 *$ & 0.117 & $0.387 *$ & 0.191 & $0.006 *$ & 0.046 & $0.005 *$ \\
\hline \multirow{4}{*}{100} & $\alpha$ & 0.536 & $9.486 *$ & 1.199 & 0.016 & 0.519 & 0.014 & 0.943 & $8.038^{*}$ & 0.466 & $7.981 *$ \\
\hline & $\lambda$ & 1.419 & $0.132 *$ & 1.593 & $0.144 *$ & 0.750 & $0.745^{*}$ & 1.475 & $0.149 *$ & 0.690 & $0.167 *$ \\
\hline & $\theta$ & 0.009 & $6.571 *$ & 0.286 & $0.682 *$ & 0.049 & $1.389 *$ & 0.024 & $0.417 *$ & $1.184 *$ & $0.134 *$ \\
\hline & $\beta$ & $1.434 *$ & $0.002 *$ & 0.343 & $0.190 *$ & 0.094 & $0.317 *$ & 0.187 & $0.006^{*}$ & 0.045 & $0.003 *$ \\
\hline \multirow{4}{*}{150} & $\alpha$ & 0.412 & $5.336 *$ & 0.828 & 0.014 & 0.224 & $6.969 *$ & 0.706 & $5.685^{*}$ & 0.222 & $4.412 *$ \\
\hline & $\lambda$ & 1.368 & $0.122 *$ & 1.483 & $0.133 *$ & 0.719 & $0.681 *$ & 1.440 & $0.113 *$ & 0.587 & $0.072 *$ \\
\hline & $\theta$ & 0.008 & $5.350 *$ & 0.259 & $0.471 *$ & 0.027 & $1.184 *$ & 0.017 & $0.302 *$ & $0.672 *$ & $0.079 *$ \\
\hline & $\beta$ & $0.672 *$ & $0.001 *$ & 0.324 & $0.129 *$ & 0.065 & $0.247 *$ & 0.180 & $0.005 *$ & 0.044 & $0.001 *$ \\
\hline \multirow{4}{*}{200} & $\alpha$ & 0.309 & $7.651 *$ & 0.561 & $7.897 *$ & 0.174 & $4.459 *$ & 0.555 & $2.845^{*}$ & 0.150 & $3.758 *$ \\
\hline & $\lambda$ & 0.976 & $0.059 *$ & 1.267 & $0.065 *$ & 0.536 & $0.304 *$ & 1.235 & $0.057 *$ & 0.466 & $0.048 *$ \\
\hline & $\theta$ & 0.005 & $4.837 *$ & 0.188 & $0.285^{*}$ & 0.009 & $0.480 *$ & $9.035 *$ & $0.148 *$ & $0.509 *$ & $0.059 *$ \\
\hline & $\beta$ & $0.459 *$ & $0.001 *$ & 0.175 & $0.056 *$ & 0.020 & $0.175^{*}$ & 0.166 & $0.002 *$ & 0.005 & $0.001 *$ \\
\hline
\end{tabular}

* Indicate that the value multiply $10^{-3}$ 
Table 5: Mean square error and estimated risk of the Bayes estimates under SE, LINEX and GE loss functions based on progressive II censoring for $(\alpha=0.25, \lambda=0.8, \theta=0.5, \beta=1)$

\begin{tabular}{|c|c|c|c|c|c|c|c|c|c|c|c|c|c|}
\hline \multirow{3}{*}{$n$} & \multirow{3}{*}{$m$} & \multirow{3}{*}{$\begin{array}{l}\text { Censored Schemes } \\
\left(R_{1}, R_{2}, R_{3}, \ldots, R_{m}\right)\end{array}$} & \multirow{3}{*}{ parameters } & \multirow{2}{*}{\multicolumn{2}{|c|}{$\begin{array}{l}\text { SE Loss } \\
\text { Function }\end{array}$}} & \multicolumn{4}{|c|}{ LINEX Loss Function } & \multicolumn{4}{|c|}{ GE Loss Function } \\
\hline & & & & & & \multicolumn{2}{|c|}{$a=-4$} & \multicolumn{2}{|c|}{$a=4$} & \multicolumn{2}{|c|}{$v=-4$} & \multicolumn{2}{|c|}{$v=4$} \\
\hline & & & & MSE & ER & MSE & ER & MSE & ER & MSE & ER & MSE & ER \\
\hline \multirow{4}{*}{30} & \multirow{4}{*}{3} & \multirow{4}{*}{$\begin{array}{c}R_{1}=7, R_{2}=R_{3}= \\
10\end{array}$} & $\alpha$ & 0.414 & $5.842 *$ & 0.845 & $6.451 *$ & 0.411 & $0.566 *$ & 0.615 & $0.976 *$ & 0.117 & $0.535^{*}$ \\
\hline & & & $\lambda$ & 0.432 & $9.453 *$ & 1.369 & $6.772 *$ & 0.272 & $0.519 *$ & 0.909 & $0.879 *$ & 0.249 & $0.053 *$ \\
\hline & & & $\theta$ & 0.167 & $4.395 *$ & 0.452 & $7.224 *$ & $7.104 *$ & $0.047 *$ & 0.502 & $0.352 *$ & $3.154 *$ & $0.087 *$ \\
\hline & & & $\beta$ & 0.543 & $8.273 *$ & 0.733 & $6.811 *$ & 0.240 & $0.094 *$ & 0.681 & $0.774 *$ & $4.095^{*}$ & $0.035 *$ \\
\hline \multirow{4}{*}{50} & \multirow{4}{*}{3} & \multirow{4}{*}{$\begin{array}{c}R_{1}=7, R_{2}=R_{3}= \\
10 .\end{array}$} & $\alpha$ & 0.392 & $2.119 *$ & 0.685 & $5.669 *$ & 0.262 & $0.433 *$ & 0.562 & $0.537 *$ & $5.349 *$ & $0.501 *$ \\
\hline & & & $\lambda$ & 0.375 & $8.327 *$ & 1.214 & $6.534 *$ & 0.251 & $0.428 *$ & 0.801 & $0.658 *$ & 0.250 & $0.046 *$ \\
\hline & & & $\theta$ & 0.139 & $4.230 *$ & 0.409 & $5.149 *$ & $6.871 *$ & $0.043 *$ & 0.367 & $0.264 *$ & $1.943 *$ & $0.078 *$ \\
\hline & & & $\beta$ & 0.439 & $4.544 *$ & 0.631 & $5.245 *$ & 0.217 & $0.061 *$ & 0.586 & $0.242 *$ & $3.554 *$ & $0.025 *$ \\
\hline \multirow{4}{*}{100} & \multirow{4}{*}{4} & \multirow{4}{*}{$\begin{array}{c}R_{1}=R_{2}=R_{3}= \\
R_{4}=24 .\end{array}$} & $\alpha$ & 0.319 & $2.008 *$ & 0.630 & $5.244 *$ & 0.234 & $0.264 *$ & 0.543 & $0.484 *$ & $4.749 *$ & $0.444 *$ \\
\hline & & & $\lambda$ & 0.233 & $3.336 *$ & 1.043 & $5.819 *$ & 0.118 & $0.221 *$ & 0.780 & $0.623 *$ & 0.123 & $0.038 *$ \\
\hline & & & $\theta$ & 0.124 & $3.927 *$ & 0.355 & $3.311 *$ & $5.258 *$ & $0.039 *$ & 0.334 & $0.159 *$ & $0.627 *$ & $0.074 *$ \\
\hline & & & $\beta$ & 0.338 & $3.717 *$ & 0.422 & $2.917 *$ & 0.161 & $0.043 *$ & 0.425 & $0.207 *$ & $2.067 *$ & $0.021 *$ \\
\hline \multirow{4}{*}{150} & \multirow{4}{*}{4} & \multirow{4}{*}{$\begin{array}{l}R_{1}=R_{3}=33 \\
R_{2}=R_{4}=40 .\end{array}$} & $\alpha$ & 0.284 & $1.867 *$ & 0.564 & $4.345^{*}$ & 0.155 & $0.185^{*}$ & 0.344 & $0.242 *$ & $4.115 *$ & $0.386 *$ \\
\hline & & & $\lambda$ & 0.147 & $3.131 *$ & 0.931 & $5.181 *$ & 0.060 & $0.164 *$ & 0.705 & $0.409 *$ & 0.020 & $0.021 *$ \\
\hline & & & $\theta$ & 0.061 & $3.379 *$ & 0.243 & $2.921 *$ & $3.022 *$ & $0.24 *$ & 0.240 & $0.135 *$ & $0.421 *$ & $0.265^{*}$ \\
\hline & & & $\beta$ & 0.215 & $2.784 *$ & 0.346 & $2.863 *$ & 0.130 & $0.034 *$ & 0.244 & $0.181 *$ & $0.604 *$ & $0.018 *$ \\
\hline \multirow{4}{*}{200} & \multirow{4}{*}{5} & \multirow{4}{*}{$\begin{array}{l}R_{1}=R_{2}=R_{3}= \\
R_{4}=R_{5}=39 .\end{array}$} & $\alpha$ & 0.042 & $1.077 *$ & 0.232 & $1.057 *$ & 0.075 & $0.159 *$ & 0.211 & $0.165 *$ & $2.038 *$ & $0.154 *$ \\
\hline & & & $\lambda$ & 0.039 & $1.445^{*}$ & 0.827 & $3.814 *$ & 0.042 & $0.144 *$ & 0.646 & $0.237 *$ & $7.472 *$ & $0.015 *$ \\
\hline & & & $\theta$ & $7.715 *$ & $1.962 *$ & 0.168 & $1.843 *$ & $2.603 *$ & $0.013 *$ & 0.197 & $0.129 *$ & $2.311 *$ & $0.015 *$ \\
\hline & & & $\beta$ & 0.091 & $2.432 *$ & 0.282 & $2.431 *$ & 0.072 & $0.011 *$ & 0.148 & $0.107 *$ & $0.111 *$ & $0.010 *$ \\
\hline
\end{tabular}

* Indicate that the value multiply $10^{-3}$

Table 6: Mean square error and estimated risk of the Bayes estimates under SE, LINEX and GE loss functions based on progressive II censoring for Censoring for $(\alpha=0.75, \lambda=1.5, \theta=0.5, \beta=1)$

\begin{tabular}{|c|c|c|c|c|c|c|c|c|c|c|c|c|c|}
\hline \multirow{3}{*}{$n$} & \multirow{3}{*}{$m$} & \multicolumn{2}{|l|}{ Censored Schemes } & \multirow{2}{*}{\multicolumn{2}{|c|}{$\begin{array}{l}\text { SE Loss } \\
\text { Function }\end{array}$}} & \multicolumn{4}{|c|}{ LINEX Loss Function } & \multicolumn{4}{|c|}{ GE Loss Function } \\
\hline & & \multirow{2}{*}{$\left(R_{1}, R_{2}, R_{3}, \ldots, R_{m}\right)$} & \multirow[t]{2}{*}{ parameters } & & & \multicolumn{2}{|c|}{$a=-4$} & \multicolumn{2}{|c|}{$a=4$} & \multicolumn{2}{|c|}{$v=-4$} & \multicolumn{2}{|c|}{$v=4$} \\
\hline & & & & MSE & ER & MSE & ER & MSE & ER & MSE & ER & MSE & ER \\
\hline \multirow{4}{*}{30} & \multirow{4}{*}{3} & \multirow{4}{*}{$\begin{array}{c}R_{1}=7, R_{2}=R_{3}= \\
10 .\end{array}$} & $\alpha$ & 0.449 & $4.203^{*}$ & 0.972 & $4.528 *$ & 0.420 & $4.589 *$ & 0.746 & $6.552 *$ & 0.127 & $5.549 *$ \\
\hline & & & $\lambda$ & 0.481 & $3.545^{*}$ & 1.589 & $7.803^{*}$ & 0.381 & $4.101 *$ & 1.107 & $9.384 *$ & 0.262 & $9.656^{*}$ \\
\hline & & & $\theta$ & 0.173 & $6.471 *$ & 0.539 & $7.288 *$ & $7.249 *$ & $7.187 *$ & 0.530 & $7.670 *$ & $3.685 *$ & $1.075^{*}$ \\
\hline & & & $\beta$ & 0.572 & $2.994 *$ & 0.751 & $3.525 *$ & 0.240 & $0.127 *$ & 0.702 & $0.342 *$ & $4.136 *$ & $0.244 *$ \\
\hline \multirow{4}{*}{50} & \multirow{4}{*}{3} & \multirow{4}{*}{$\begin{array}{c}R_{1}=7, R_{2}=R_{3}= \\
10 .\end{array}$} & $\alpha$ & 0.436 & $3.289 *$ & 0.734 & $4.065 *$ & 0.296 & $2.542 *$ & 0.652 & $3.076 *$ & $5.677 *$ & $5.057 *$ \\
\hline & & & $\lambda$ & 0.405 & $2.563 *$ & 1.463 & $6.484 *$ & 0.271 & $2.132 *$ & 0.994 & $3.579 *$ & 0.169 & $6.583 *$ \\
\hline & & & $\theta$ & 0.154 & $5.622 *$ & 0.500 & $4.727 *$ & $7.054 *$ & $5.401 *$ & 0.481 & $3.637 *$ & $2.027 *$ & $0.889 *$ \\
\hline & & & $\beta$ & 0.468 & $2.106^{*}$ & 0.700 & $1.132 *$ & 0.228 & $0.082 *$ & 0.596 & $0.311 *$ & $4.371 *$ & $0.078 *$ \\
\hline \multirow{4}{*}{100} & \multirow{4}{*}{4} & \multirow{4}{*}{$\begin{array}{c}R_{1}=R_{2}=R_{3}= \\
R_{4}=24 .\end{array}$} & $\alpha$ & 0.362 & $2.868 *$ & 0.682 & $3.941 *$ & 0.259 & $1.989 *$ & 0.565 & $2.900 *$ & $5.097 *$ & $4.068 *$ \\
\hline & & & $\lambda$ & 0.283 & $2.182 *$ & 1.409 & $5.321 *$ & 0.129 & $1.320 *$ & 0.867 & $2.757^{*}$ & 0.109 & $2.491 *$ \\
\hline & & & $\theta$ & 0.129 & $5.558 *$ & 0.429 & $3.154 *$ & $5.804 *$ & $3.008 *$ & 0.380 & $2.558 *$ & $0.609 *$ & $0.531 *$ \\
\hline & & & $\beta$ & 0.445 & $1.353 *$ & 0.538 & $7.642 *$ & 0.195 & $0.048 *$ & 0.462 & $0.288^{*}$ & $3.440 *$ & $0.064 *$ \\
\hline \multirow{4}{*}{150} & \multirow{4}{*}{4} & \multirow{4}{*}{$\begin{array}{l}R_{1}=R_{3}=33, \\
R_{2}=R_{4}=40 .\end{array}$} & $\alpha$ & 0.336 & $2.489 *$ & 0.610 & $1.379 *$ & 0.181 & $1.157 *$ & 0.412 & $2.675 *$ & $4.256^{*}$ & $3.013 *$ \\
\hline & & & $\lambda$ & 0.200 & $2.101 *$ & 1.254 & $4.477^{*}$ & 0.026 & $0.977 *$ & 0.840 & $1.592 *$ & 0.020 & $1.614 *$ \\
\hline & & & $\theta$ & 0.085 & $4.639 *$ & 0.313 & $3.023 *$ & $4.471 *$ & $2.841 *$ & 0.354 & $1.866 *$ & $0.512 *$ & $0.373 *$ \\
\hline & & & $\beta$ & 0.238 & $0.984 *$ & 0.516 & $5.488 *$ & 0.169 & $0.046^{*}$ & 0.259 & $0.220 *$ & $0.623 *$ & $0.038 *$ \\
\hline \multirow{4}{*}{200} & \multirow{4}{*}{5} & \multirow{4}{*}{$\begin{array}{c}R_{1}=R_{2}=R_{3}= \\
R_{4}=R_{5}=39 .\end{array}$} & $\alpha$ & 0.074 & $1.868 *$ & 0.270 & $1.126^{*}$ & 0.084 & $1.144^{*}$ & 0.282 & $1.008 *$ & $2.573 *$ & $1.790 *$ \\
\hline & & & $\lambda$ & 0.064 & $1.556^{*}$ & 1.152 & $1.510 *$ & 0.023 & $0.754^{*}$ & 0.682 & $1.302 *$ & $7.762 *$ & $1.345^{*}$ \\
\hline & & & $\theta$ & $7.851 *$ & $2.748 *$ & 0.228 & $2.656 *$ & $3.692 *$ & $1.662 *$ & 0.237 & $1.207 *$ & $0.309 *$ & $0.129 *$ \\
\hline & & & $\beta$ & 0.159 & $0.453^{*}$ & 0.510 & $3.525^{*}$ & 0.101 & $0.028 *$ & 0.172 & $0.159 *$ & $0.145^{*}$ & $0.025^{*}$ \\
\hline
\end{tabular}

* Indicate that the value multiply $10^{-3}$

\section{Conclusions}

This study deals with the Bayesian estimation problem based on GOS from EWP distribution. For Bayesian estimates, the performance depends on the form of the prior distribution, and the loss function assumed. Most authors used squared error as symmetric loss function. However, in practice, the real loss function is often not symmetric. Therefore, the Bayesian estimators of unknown parameters of EWP distribution are obtained under symmetric (squared error) and asymmetric (LINEX and general entropy) loss functions. Additionally, the Bayes estimates based on progressive type II censored samples and joint density function of ordinary order statistics are derived as special cases. Performances of the estimator are evaluated through their MSE and ER.

Simulation study revealed that the MSE of the Bayes estimates for the first set of parameters is smaller than the MSE of the Bayes estimates of the second set of parameters for all sample sizes under GOS, joint density function of 
ordinary order statistics and progressive type II censored. While, ER of the second set of parameters is smaller than ER of the first set of parameters for all sample sizes under GOS, joint density function of ordinary order statistics and progressive type II censored data. The ER and MSE of all different estimators decrease as sample sizes increase. Under GE loss function, MSE of Bayes estimates has the smallest values as compared with the corresponding Bayes estimates under LINEX and SE loss functions. MSE of the Bayes estimates under GE loss function with parameter $v=4$ based on progressively type II censored is smaller than MSE of Bayes estimates under GOS and the joint density function of ordinary order statistics. In most cases, Bayesian estimator under general entropy based on progressive type II censored has the smallest mean squared error based on GOS and the joint density function of ordinary order statistics.

\section{References}

[1] Abd-Elfattah, A. M., Hassan, A. S., and Nassr, S. G. (2013), Maximum likelihood estimation of the parameters for exponentiated Weibull Poisson distribution based on generalized order statistics, Proceeding of the 48th Annual Conference of Statistics, Computer Sciences and Operation Research, ISSR, Cairo University, 40-56.

[2] Abd-Ellah, A. H. (2012), Bayesian and non-Bayesian estimation of the inverse Weibull model based on generalized order statistics, Intelligent Information Management, 4, 23 - 31. http://dx.doi.org/10.4236/iim.2012.42004

[3] Aboeleneen, Z. A. (2010), Inference for Weibull distribution under generalized order statistics, Mathematics and Computers in Simulation, 8, 26 - 36. http://dx.doi.org/10.1016/j.matcom.2010.06.013.

[4] Abo-Elfotouh, S., and Nassar, M. M. (2011), Estimation for the parameters of the Weibull extension model based on generalized order statistics, International Journal of Contemporary Mathematical Sciences, 6 (36), 1749 - 1760.

[5] Ahsanullah, M. (1996), Generalized order statistics from two parameter uniform distribution, Communication in Statistics - Theory and Methods, 25 (10), $2311-2318$.

[6] Ahsanullah, M. (2000), Generalized order statistics from exponential distribution, Journal of Statistical Planning and Inference, 85 , 85 - 91. http://dx.doi.org/10.1016/S0378-3758(99)00068-3.

[7] Ateya, S. F. (2013), Estimation under modified Weibull distribution based on right censored generalized order statistics, Journal of Applied Statistics, 4 (12), 2720 - 2734. http://dx.doi.org/10.1080/02664763.2013.825705.

[8] Balakrishnan, N., and Aggarwala, R. (2000), Progressive Censored-Theory, Methods, and Application, Birkhauser, Boston.

[9] Basu, A. P., and Ibrahimi, N. (1991), Bayesian approach to life testing and reliability estimating using asymmetric loss function, Journal of Statistical Planning and Inference, 29, 21 - 31. http://dx.doi.org/10.1016/0378-3758(92)90118-C.

[10] Burkschat, M., Cramer, E., and Kamps, U. (2007), Linear estimation of location and scale parameters based on generalized order statistics from generalized Pareto distributions, In: M. Ahsanullah, Ed., recent Developments in Ordered Random Variables, Nova Science Publisher, New York, and PP. 253 - 261.

[11] Burkschat, M. (2010), Linear estimators and predictors based on generalized order statistics from generalized Pareto distributions, Communication in Statistics - Theory and Methods, 39 (2), 311 - 326.

[12] Calabria, R., and Pulcini, G. (1996), Point estimation under asymmetric loss function for left truncated exponential samples, Communication in Statistics - Theory and Methods, 25 (3), $285-600$.

[13] Dey, D. K., Ghosh, M. and Srinivasan, C. (1987), Simultaneous estimation of parameters under entropy loss, J. Statist. Plann. Inference, 25, $347-363$.

[14] Dey, D. K., and Liu, P. L. (1992), On comparison of estimators in a generalized life model, Microelectron Reliab., 32, 207 - 221. http://dx.doi.org/10.1016/0026-2714(92)90099-7.

[15] Habibullah, M., and Ahsanullah. M. (2000), Estimation of parameters of a Pareto distribution by generalized order statistics, Communication in Statistics - Theory and Methods, 29 (7), 1597 - 1609.

[16] Jaheen, Z. F. (2005), Estimation based on generalized order statistics from the Burr model, Communication in Statistics - Theory and Methods, 34, $785-794$

[17] Kamps, U. (1995), A concept of generalized order statistics, Journal of Statistical Planning and Inference, 48 , 1 - 23. http://dx.doi.org/10.1016/0378-3758(94)00147-N.

[18] Kim, C., and Han, K. (2014), Bayesian estimation of Rayleigh distribution based on generalized order statistics, Applied Mathematics Sciences, 8 (150), $7475-7485$.

[19] Koch, K. R. (2007), Introduction to Bayesian Statistics, Second edition, Berlin, Springer.

[20] Lindley, D. V. (1980), Approximate Bayesian method, Trabajos de Estadistica, 31, 223 - 237. http://dx.doi.org/10.1007/BF02888353.

[21] Mahmoudi, E. and Sepahdar, A. (2012), Exponentiated Weibull-Poisson distribution: model, properties and applications, Mathematics and Computers in Simulation, 92, 76 - 97. http://dx.doi.org/10.1016/j.matcom.2013.05.005.

[22] Mailinowska. I., Pawlas, P., and Szynal, D. (2006), Estimation of location and scale parameters for the Burr XII using generalized order statistics, Linear Algebra and its Applications, 417, 150 - 162. http://dx.doi.org/10.1016/j.laa.2006.02.007.

[23] Moghadam, M. S., Yaghmaei, F., and Babanezhad, M. (2012), Inference for Lomax distribution under generalized order statistics, Applied Mathematical Sciences, 6 (105), 5241 - 5251.

[24] Safi, S. K., and Ahmed, R. H. (2013), Statistical estimation based on generalized order statistics from Kumaraswamy distribution, Proceeding of the 14st Applied Stochastic Models and Data Analysis (ASMDA)International Conference, Mataro (Barcelona), Spain, 25-28.

[25] Singh, U., Gupta, P. K., and Upadhyay, S. K. (2005), Estimation of three parameter exponentiated Weibull distribution under type II censoring, Journal of Statistical Planning and Inference, 134, 350 - 372. http://dx.doi.org/10.1016/j.jspi.2004.04.018.

[26] Varian, H. R. (1975), A Bayesian approach to real estate assessment, In Studies in Bayesian Econometrics and Statistics in Honour of Leonard J. Savage, eds. Stephen E. Fienberg and Arnold Zellner, Amsterdam: North Holland. Pp. 195 - 208.

[27] Zellner, A. (1986), Bayesian estimation and prediction using asymmetric loss function, Journal of the American Statistical Associations, 81 (394), 446 - 451. http://dx.doi.org/10.1080/01621459.1986.10478289. 